\title{
铁(III)催化亚胺和邻苯二胺的有氧氧化合成苯并咪唑的研究
}

\author{
赵丹丹虞家涛王鹏程陆 明* \\ (南京理工大学化工学院 江苏 南京 210094)
}

\begin{abstract}
摘要 详细探究了铁催化氧化亚胺和邻苯二胺生成苯并咪唑的合成反应. 该反应过程可分为三步：第一步是亚胺和邻 苯二胺氧化缩合生成席夫碱，第二步是席夫碱与邻苯二胺上另一氨基亲核加成生成苯并咪唑啉，第三步苯并咪唑啉氧 化脱氢生成目标产物苯并咪唑. 结果表明, 以 $\mathrm{Fe}\left(\mathrm{NO}_{3}\right)_{3} \cdot 9 \mathrm{H}_{2} \mathrm{O}$ 为催化剂, 乙腈为溶剂, 在 $80{ }^{\circ} \mathrm{C}$ 下应 $10 \mathrm{~h}$ 可以获得良 好收率. 而且这种方法以经济、绿色的空气作为氧化剂, 具有良好的应用前景.

关键词 苯并咪唑衍生物; 合成; $\mathrm{Fe}\left(\mathrm{NO}_{3}\right)_{3} \cdot 9 \mathrm{H}_{2} \mathrm{O}$; 亚胺; 邻苯二胺
\end{abstract}

\section{Aerobic Oxidation Synthesis of Benzimidazoles via Iron(III)-Catalyzed the Reaction of Imine Derivatives with o-Phenylenediamine}

\author{
Zhao, Dandan Yu, Jiatao Wang, Pengcheng Lu, Ming* \\ (College of Chemical Engineering, Nanjing University of Science and Technology, Nanjing 210094)
}

\begin{abstract}
In this paper, Iron(III)-catalyzed aerobic oxidation of imine derivatives and $o$-phenylenediamine under air condition is studied in detail. With $\mathrm{Fe}\left(\mathrm{NO}_{3}\right)_{3} \cdot 9 \mathrm{H}_{2} \mathrm{O}$ as catalyst and air as green and economical oxidant, the reaction could take place in acetonitrile at $80{ }^{\circ} \mathrm{C}$ and affords benzimidazoles as target compounds in good yields after $10 \mathrm{~h}$, exhibiting good application prospect as well. This process includes three steps: (1) the aerobic oxidative condensation of imine and $o$-phenylenediamine to form Schiff base, (2) the formation of benzimidazoline via nucleophilic addition of Shift base and amine group on the $o$-phenylenediamine, and (3) the dehydration of benzimidazoline to form benzimidazole as target compounds.
\end{abstract}

Keywords benzimidazoles; synthesis; $\mathrm{Fe}\left(\mathrm{NO}_{3}\right)_{3} \cdot 9 \mathrm{H}_{2} \mathrm{O}$; imine derivatives; $o$-phenylenediamine

苯并咪唑类化合物是一种含有两个氮原子的杂环 化合物 ${ }^{[1,2]}$, 由于其结构特性、生理功效、反应活性和优 良的配位性能而具有多种生物生理活性. 苯并咪唑及其 衍生物具有驱虫、杀菌、抗癌等药理学功效 ${ }^{[3]}$, 同时在 高能量密度化合物、高性能复合材料、金属防腐蚀、感 光材料等诸多领域也有广泛应用, 因此, 苯并咪唑类化 合物的合成具有重大的现实意义. 通常情况下该类化合 物有两种合成途径 ${ }^{[4]}$. 一是采用邻苯二胺与羧酸及其衍 生物为合成原料, 二是采用邻苯二胺与醛为合成原料. 但是这两种方法都有一定的局限性. 前者需要在强酸和 高温的条件下进行, 后者需要加入化学计量的 $\left(\mathrm{NH}_{4}\right)_{2} \mathrm{~S}_{2} \mathrm{O}_{8}{ }^{[5]}, \mathrm{H}_{2} \mathrm{O}_{2}{ }^{[6]}$ 等氧化剂. 生成的副产物, 不仅给 后续化合物的分离造成困难, 还会危害环境.

铁由于价格低廉、生物兼容性好等特点, 成为人们 努力发展的新一代催化剂. 最近几年, 铁催化的氢化反
应成为研究热点 ${ }^{[7]} .2014$ 年, $\mathrm{Guo}$ 等 ${ }^{[8]}$ 将铁负载在 $\mathrm{SiO}_{2}$ 上, 实现了甲烷的无氧直接转化, 得到产物乙烯、芳基 化合物和氢气. 2014 年, $\mathrm{Li}$ 等 ${ }^{[9]}$ 研发出新型手性二十二 元环状胺膦配体与 $\mathrm{Fe}_{3}(\mathrm{CO})_{12}$ 原位生成的催化体系, 催 化芳香酮、杂环芳香酮和 $\beta$-酮酯的不对称氢化反应. 同 年, Shen 等 ${ }^{[10]}$ 通过使用手性螺环双噁唑啉为配体, 实现 了铁高效催化分子内的不对称环丙化反应. Zhang 等 ${ }^{[1]}$ 以 $\mathrm{FeCl}_{3}$ 作为催化剂, 在温和的条件下实现了叔胺氮在 邻位碳原子上 $\mathrm{C}-\mathrm{H}$ 键与亚磷酸二烷基酯等的 $\mathrm{H}-\mathrm{P}$ 键 交叉脱氢偶联. $\mathrm{Liu}$ 等 ${ }^{[12]}$ 以廉价、低毒的 $\mathrm{Fe}(\mathrm{OAc})_{2}$ 为催 化剂, 在三氟乙酸助催化下, 实现了 2-甲基氮杂芳烃与 芳香酫的加成脱水反应，选择性合成具有生物活性的反 式 2-烯基氮杂芳烃化合物. 2015 年, Guo 等 ${ }^{[13]}$ 以 $\mathrm{FeCl}_{2}$ 为催化剂, 膦烷基联吡啶为配体, $\mathrm{LiAlH}_{4}$ 为还原剂, 实 现了烯烃的氢化反应.

\footnotetext{
*E-mail: luming@mail.njust.edu.cn

Received July 14, 2015; revised September 10, 2015; published online September 17, 2015

Project supported by the National Natural Science Foundation of China (No. 51374131).

国家自然科学基金(No. 51374131)资助项目.
} 
在我们课题组先前的工作中, 已经完成了在空气氛 围中, 通过醇或胺和邻苯二胺反应生成苯并咪唑 ${ }^{[14 ~ 16]}$. 现在采用更加简单、高效的有氧氧化体系, 即以 $\mathrm{Fe}\left(\mathrm{NO}_{3}\right)_{3} \cdot 9 \mathrm{H}_{2} \mathrm{O}$ 为催化剂, 通过亚胺和邻苯二胺氧化缩 合生成苯并咪唑, 如 Scheme 1 所示. 该方法与文献报道 方法相比有以下优越性: 首先, 用铁作为催化剂, 价格 低廉; 其次, 用亚胺代替醛作为反应原料, 具有稳定, 低毒等优点; 最后, 使用绿色、经济的空气作为氧化剂, 反应条件温和, 副产物为水, 简单、环保、实用. 因此, 铁催化亚胺与邻苯二胺氧化缩合制备苯并咪唑的方法, 具有研究意义和应用前景.

先前的工作
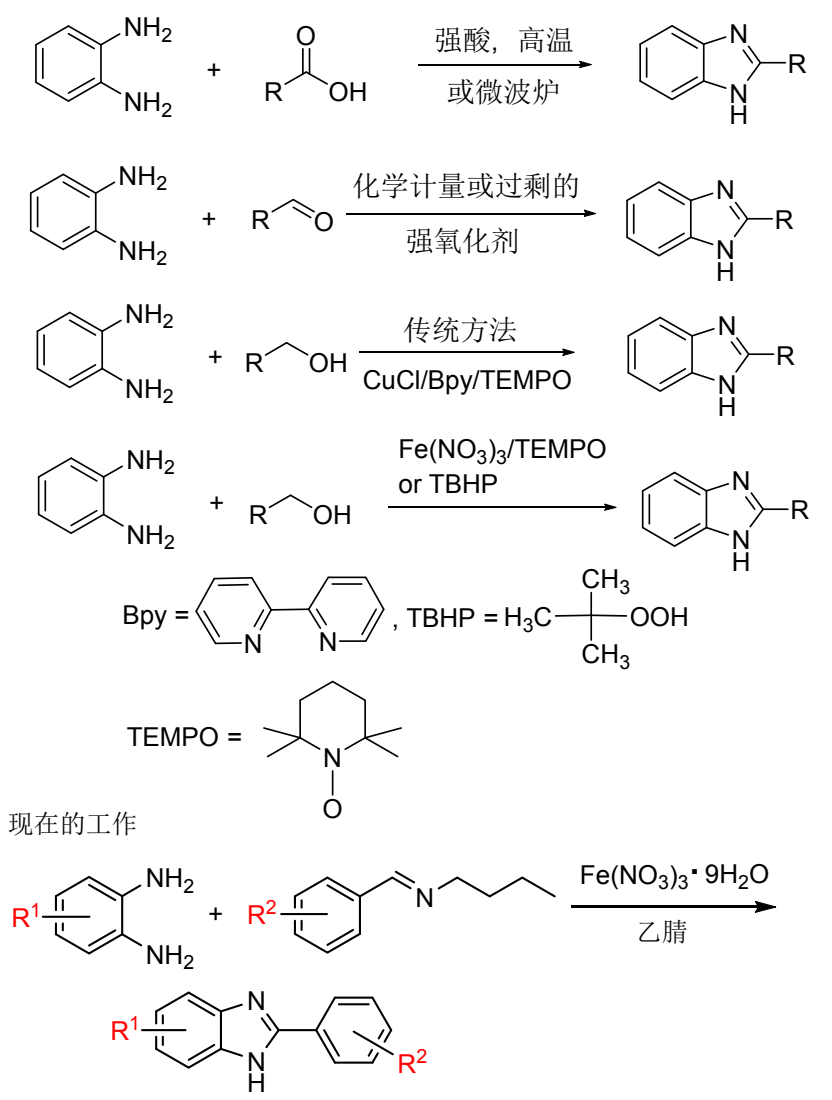

图式 1 苯并咪唑的合成方法

Scheme 1 Methods for the preparing the benzimidazoles

\section{1 结果与讨论}

\section{1 反应条件的优化}

在初始的实验中, 以邻苯二胺和 $N$-亚苄基丁胺的 反应作为模板, 进行反应条件的筛选, 主要考察催化剂 的种类、反应温度、催化剂用量、反应物用量、气体氛 围和溶剂对模板反应的影响，其实验结果见表 1 .

首先, 考察了催化剂种类对反应收率的影响. 在没 有催化剂的条件下, 邻苯二胺和亚胺在 $80{ }^{\circ} \mathrm{C}$ 、空气氛
围、乙腈溶剂中反应，仅可以得到少量的目标产物(表 1, Entry 1). 当加入 $5 \mathrm{~mol} \% \mathrm{Fe}\left(\mathrm{NO}_{3}\right)_{3} \cdot 9 \mathrm{H}_{2} \mathrm{O}$, 收率可以达 到 78\%(表 1, Entry 2), 除此之外, 还采用了 $\mathrm{FeCl}_{3}$, $\mathrm{Fe}_{2}\left(\mathrm{SO}_{4}\right)_{3}$ 作为催化剂, 也取得了不错的收率(表 1 , Entries 3，4). 但是当采用别的金属盐，比如 $\mathrm{Cu}(\mathrm{OAc})_{2}$, $\mathrm{CuCl}_{2}, \mathrm{Cu}\left(\mathrm{NO}_{3}\right)_{2}, \mathrm{CuI}$ 和 $\mathrm{CoCl}_{2}$ 作为催化剂时, 则目标产 物的收率较低(表 1, Entries 5 9). 和以上催化剂相比, $\mathrm{Fe}\left(\mathrm{NO}_{3}\right)_{3} \cdot 9 \mathrm{H}_{2} \mathrm{O}$ 具有更好的催化活性. 其次, 考察了反 应物用量对收率的影响. 当邻苯二胺采用 1.5 equiv 时, 目标产物收率最好，可以达到 86\%(表 1, Entries 2,10 13). 再次, 考察了催化剂的用量对反应影响. 当 $\mathrm{Fe}\left(\mathrm{NO}_{3}\right)_{3} \cdot 9 \mathrm{H}_{2} \mathrm{O}$ 的用量为 $5 \mathrm{~mol} \%$ 时，收率为 $86 \%$ (表 1, Entries 11, 14 15). 溶剂也是影响目标产物收率的重要 因素, 将乙醇、DMSO、甲苯、乙腈四种溶剂进行对比, 发现乙腈更利于反应的发生(表 1, Entries 11，16１8). 然后又考察了温度对收率的影响. 起初产率随着温度的 提高而增大, 但是, 当温度达到 $80{ }^{\circ} \mathrm{C}$ 时, 温度再提高, 产率反而降低(表 1, Entries 11，19２1). 最后，考察了 气体氛围的影响. 处于氧气或者空气氛围中, 收率较高, 处于 $\mathrm{N}_{2}$ 氛围下, 仅可以得到少量的目标产物(表 1, Entries 11, 22 23).

\section{2 底物取代基对化学反应的影响}

在获得最佳反应条件后, 为了充分评价底物适用范 围，论文探讨了带有不同取代基团的亚胺和邻苯二胺发 生化学反应的情况, 研究结果如表 2 所示. 首先, 考察 不同伯胺的反应性. 正丁胺、苯胺、茮胺、叔丁胺衍生 的亚胺都具有很高的活性(表 2, Entry 1). 其次, 考察正 丁胺衍生的亚胺上取代基的影响. 当亚胺苯环上连有吸 电子基 $\mathrm{NO}_{2}, \mathrm{Cl}, \mathrm{Br}$ 时, 得到较高的反应收率(表 2, Entries 1,3 7). 因为 $\mathrm{NO}_{2}, \mathrm{Cl}, \mathrm{Br}$ 的吸电子作用使得 $\mathrm{C}=\mathrm{N}$ 电子云密度下降, 更有利于邻苯二胺 $\mathrm{N}$ 上的孤对电子去 进攻 $\mathrm{C}=\mathrm{N}$ 上的 $\mathrm{C}$ 发生亲核加成. $\mathrm{Cl}$ 和 $\mathrm{Br}$ 是邻对位定位 基, 所以亚胺苯环上 2,4 位连有 $\mathrm{Cl}$ 的收率最高. $\mathrm{NO}_{2}$ 是 间位定位基, 所以亚胺苯环上间位连有 $\mathrm{NO}_{2}$ 的收率也较 高. 当亚胺苯环上连有供电子基时, 收率较低, 且供电 子基越多, 供电子能力越强, 位阻越大, 收率越低(表 2 , Entries $1,2,8,9)$. 当亚胺的芳环为萗环时, 反应也能得 到较好收率(表 2, Entry 10). 再次, 考察邻苯二胺上取代 基的影响. 当邻苯二胺上连有硝基或氯时，和无取代基 相比，收率均下降(表 2, Entry 1,11). 最后，考察杂芳醛 的影响。选用吲哚甲醛，目标产物收率为 $72 \%$ (表 2 , Entry 12); 选用吡啶甲醛，目标产物收率为 $78 \%$ (表 2, Entry 13). 对反应底物进行充分拓展，目标产物均取得 了不错的收率，充分证明了通过亚胺和邻苯二胺这种方 法合成苯并咪唑的优越性. 
表 1 铁催化亚胺和邻苯二胺的反应条件优化 ${ }^{a}$

Table 1 Optimization of reaction conditions in the iron-catalyzed coupling of imine derivatives and $o$-phenylenediamine

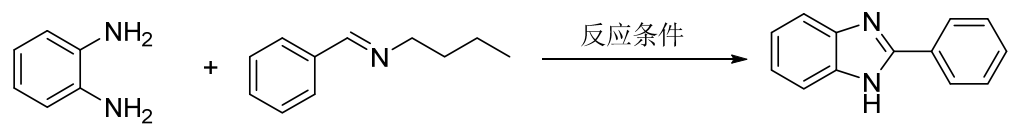

\begin{tabular}{|c|c|c|c|c|c|c|}
\hline Entry & $o$-Phenylenediamine/equiv. & Catalyst (mol\%) & Solvent & $t /{ }^{\circ} \mathrm{C}$ & Gas & Yield $^{b} / \%$ \\
\hline 1 & 1.0 & - & $\mathrm{MeCN}$ & 80 & Air & Trace \\
\hline 2 & 1.0 & $\mathrm{Fe}\left(\mathrm{NO}_{3}\right)_{3} \cdot 9 \mathrm{H}_{2} \mathrm{O}(5)$ & $\mathrm{MeCN}$ & 80 & Air & 78 \\
\hline 3 & 1.0 & $\mathrm{FeCl}_{3}(5)$ & $\mathrm{MeCN}$ & 80 & Air & 77 \\
\hline 4 & 1.0 & $\mathrm{Fe}_{2}\left(\mathrm{SO}_{4}\right)_{3}(5)$ & $\mathrm{MeCN}$ & 80 & Air & 73 \\
\hline 5 & 1.0 & $\mathrm{Cu}(\mathrm{OAc})_{2}(5)$ & $\mathrm{MeCN}$ & 80 & Air & 53 \\
\hline 6 & 1.0 & $\mathrm{CuCl}_{2}(5)$ & $\mathrm{MeCN}$ & 80 & Air & 55 \\
\hline 7 & 1.0 & $\mathrm{Cu}\left(\mathrm{NO}_{3}\right)_{2}(5)$ & $\mathrm{MeCN}$ & 80 & Air & 58 \\
\hline 8 & 1.0 & $\mathrm{CuI}(5)$ & $\mathrm{MeCN}$ & 80 & Air & 65 \\
\hline 9 & 1.0 & $\mathrm{CoCl}_{2}(5)$ & $\mathrm{MeCN}$ & 80 & Air & 42 \\
\hline 10 & 1.2 & $\mathrm{Fe}\left(\mathrm{NO}_{3}\right)_{3} \cdot 9 \mathrm{H}_{2} \mathrm{O}(5)$ & $\mathrm{MeCN}$ & 80 & Air & 80 \\
\hline 11 & 1.5 & $\mathrm{Fe}\left(\mathrm{NO}_{3}\right)_{3} \cdot 9 \mathrm{H}_{2} \mathrm{O}(5)$ & $\mathrm{MeCN}$ & 80 & Air & 86 \\
\hline 12 & 0.8 & $\mathrm{Fe}\left(\mathrm{NO}_{3}\right)_{3} \cdot 9 \mathrm{H}_{2} \mathrm{O}(5)$ & $\mathrm{MeCN}$ & 80 & Air & 70 \\
\hline 13 & 2.0 & $\mathrm{Fe}\left(\mathrm{NO}_{3}\right)_{3} \cdot 9 \mathrm{H}_{2} \mathrm{O}(5)$ & $\mathrm{MeCN}$ & 80 & Air & 43 \\
\hline 14 & 1.5 & $\mathrm{Fe}\left(\mathrm{NO}_{3}\right)_{3} \cdot 9 \mathrm{H}_{2} \mathrm{O}$ & $\mathrm{MeCN}$ & 80 & Air & 71 \\
\hline 15 & 1.5 & $\mathrm{Fe}\left(\mathrm{NO}_{3}\right)_{3} \cdot 9 \mathrm{H}_{2} \mathrm{O}(10)$ & $\mathrm{MeCN}$ & 80 & Air & 85 \\
\hline 16 & 1.5 & $\mathrm{Fe}\left(\mathrm{NO}_{3}\right)_{3} \cdot 9 \mathrm{H}_{2} \mathrm{O}(5)$ & $\mathrm{EtOH}$ & 80 & Air & 83 \\
\hline 17 & 1.5 & $\mathrm{Fe}\left(\mathrm{NO}_{3}\right)_{3} \cdot 9 \mathrm{H}_{2} \mathrm{O}(5)$ & DMSO & 80 & Air & 58 \\
\hline 18 & 1.5 & $\mathrm{Fe}\left(\mathrm{NO}_{3}\right)_{3} \cdot 9 \mathrm{H}_{2} \mathrm{O}(5)$ & Toluene & 80 & Air & 77 \\
\hline 19 & 1.5 & $\mathrm{Fe}\left(\mathrm{NO}_{3}\right)_{3} \cdot 9 \mathrm{H}_{2} \mathrm{O}(5)$ & $\mathrm{MeCN}$ & 25 & Air & Trace \\
\hline 20 & 1.5 & $\mathrm{Fe}\left(\mathrm{NO}_{3}\right)_{3} \cdot 9 \mathrm{H}_{2} \mathrm{O}(5)$ & $\mathrm{MeCN}$ & 50 & Air & 49 \\
\hline 21 & 1.5 & $\mathrm{Fe}\left(\mathrm{NO}_{3}\right)_{3} \cdot 9 \mathrm{H}_{2} \mathrm{O}(5)$ & $\mathrm{MeCN}$ & 100 & Air & 43 \\
\hline 22 & 1.5 & $\mathrm{Fe}\left(\mathrm{NO}_{3}\right)_{3} \cdot 9 \mathrm{H}_{2} \mathrm{O}(5)$ & $\mathrm{MeCN}$ & 80 & $\mathrm{O}_{2}$ & 86 \\
\hline 23 & 1.5 & $\mathrm{Fe}\left(\mathrm{NO}_{3}\right)_{3} \cdot 9 \mathrm{H}_{2} \mathrm{O}(5)$ & $\mathrm{MeCN}$ & 80 & $\mathrm{~N}_{2}$ & 5 \\
\hline
\end{tabular}

反应条件: 亚胺, 邻苯二胺, 催化剂, 溶剂 $(5 \mathrm{~mL})$, 特定温度, 气体氛围, $10 \mathrm{~h} ;{ }^{b}$ 分离产率.

\section{3 反应机理}

我们推测反应过程可分为三步: 第一步, 亚胺和邻 苯二胺氧化缩合生成席夫碱; 第二步, 席夫碱与邻苯二 胺上另一氨基发生亲核加成生成苯并咪唑啉; 第三步, 苯并咪唑啉氧化脱氢生成目标产物苯并咪唑. 首先关注 第一步, 以 $N$-亚芳基丁胺和苯胺为原料合成了 $N$-苯亚 甲基苯胺, 见表 3. 当 $N$-亚苄基丁胺和苯胺的物质的量 比为 $1: 1.5, \mathrm{Fe}\left(\mathrm{NO}_{3}\right)_{3} \cdot 9 \mathrm{H}_{2} \mathrm{O} 5 \mathrm{~mol} \%, 80{ }^{\circ} \mathrm{C}$. 乙腈作为 溶剂, 在空气氛围下反应 $10 \mathrm{~h}$, 收率可以达到 $90 \%$ (表 3, Entry 1). 但当缺少 $\mathrm{Fe}\left(\mathrm{NO}_{3}\right)_{3} \cdot 9 \mathrm{H}_{2} \mathrm{O}$ 或在 $\mathrm{N}_{2}$ 存在下, 仅 可以得到痕量的目标产物亚胺 (表 3, Entries 2,3). 因此, 反应第一步为有氧氧化步骤, 需要空气作为氧化剂, $\mathrm{Fe}\left(\mathrm{NO}_{3}\right)_{3} \cdot 9 \mathrm{H}_{2} \mathrm{O}$ 作为催化剂. 在以上结果的基础上, 探 究苯并咪唑啉的合成. 苯并咪唑啉由当量的邻苯二胺和 苯甲醛在室温条件下, 于混合溶剂中 $\left[V\left(\mathrm{H}_{2} \mathrm{O}\right)\right.$ : $\left.V\left(\mathrm{C}_{2} \mathrm{H}_{5} \mathrm{OH}\right)=1: 1\right]$ 反应 $2 \mathrm{~h}$ 制得, 但是苯并咪唑啉在室 温条件下不能稳定存在. 最后探究苯并咪唑啉的环化脱 氢, 初步探究结果见表 4 . 当 $\mathrm{Fe}\left(\mathrm{NO}_{3}\right)_{3} \cdot 9 \mathrm{H}_{2} \mathrm{O}$ 和空气均存 在的条件下, 目标产物的收率可以达到 $88 \%$ (表 4, Entry
1). 当缺少 $\mathrm{Fe}\left(\mathrm{NO}_{3}\right)_{3} \cdot 9 \mathrm{H}_{2} \mathrm{O}$ 时, 苯并咪唑收率大幅下降, 仅为 $24 \%$ (表 4, Entry 2). 在 $\mathrm{N}_{2}$ 氛围下, 仅可以得到痕量 的目标产物(表 4, Entry 3).

考虑到 $\mathrm{Fe}\left(\mathrm{NO}_{3}\right)_{3} \cdot 9 \mathrm{H}_{2} \mathrm{O}$ 作为 Lewis 酸催化丁胺的离 去确实很有可能发生, 我们做了一个实验, 见表 3 . 发 现在空气氛围, $\mathrm{Fe}\left(\mathrm{NO}_{3}\right)_{3} \cdot 9 \mathrm{H}_{2} \mathrm{O}$ 存在条件下, 亚胺收率为 $90 \%$; 在有空气, 无 $\mathrm{Fe}\left(\mathrm{NO}_{3}\right)_{3} \cdot 9 \mathrm{H}_{2} \mathrm{O}$ 的条件下, 亚胺收率 为痕量; 而当有 $\mathrm{Fe}\left(\mathrm{NO}_{3}\right)_{3} \cdot 9 \mathrm{H}_{2} \mathrm{O}, \mathrm{N}_{2}$ 氛围下, 亚胺收率也 为痕量, 所以反应过程中需要空气和 $\mathrm{Fe}\left(\mathrm{NO}_{3}\right)_{3} \cdot 9 \mathrm{H}_{2} \mathrm{O}$ 两 者的共同存在. 因此, 我们认为反应机理图 1 中亚胺 1 到席夫碱 3 的过程更有可能为 $\mathrm{Fe}\left(\mathrm{NO}_{3}\right)_{3} \cdot 9 \mathrm{H}_{2} \mathrm{O}$ 催化的有 氧氧化过程. 席夫碱 3 由于 $\mathrm{N}$ 上孤对电子的存在, 会发 生分子内的加成反应, 生成苯并咪唑啉中间体 4. 在 $\mathrm{Fe}(\mathrm{III}), \mathrm{O}_{2}$ 存在条件下, 中间体 4 会发生环化脱氢生成目 标产物 5.

\section{2 结论}

本文一方面探究了铁催化氧化亚胺和邻苯二胺生 成苯并咪唑的合成条件. 即当亚胺和邻苯二胺的物质的 量比为 $1: 1.5, \mathrm{Fe}\left(\mathrm{NO}_{3}\right)_{3} \cdot 9 \mathrm{H}_{2} \mathrm{O} 5 \mathrm{~mol} \%, 80{ }^{\circ} \mathrm{C}$. 乙腈作 
表 2 亚胺对反应的影响 ${ }^{a}$

Table 2 Effect of imines on the reaction

$$
\text { 乙腈, } 80^{\circ} \mathrm{C}, 10 \mathrm{~h}, \mathrm{Air}
$$

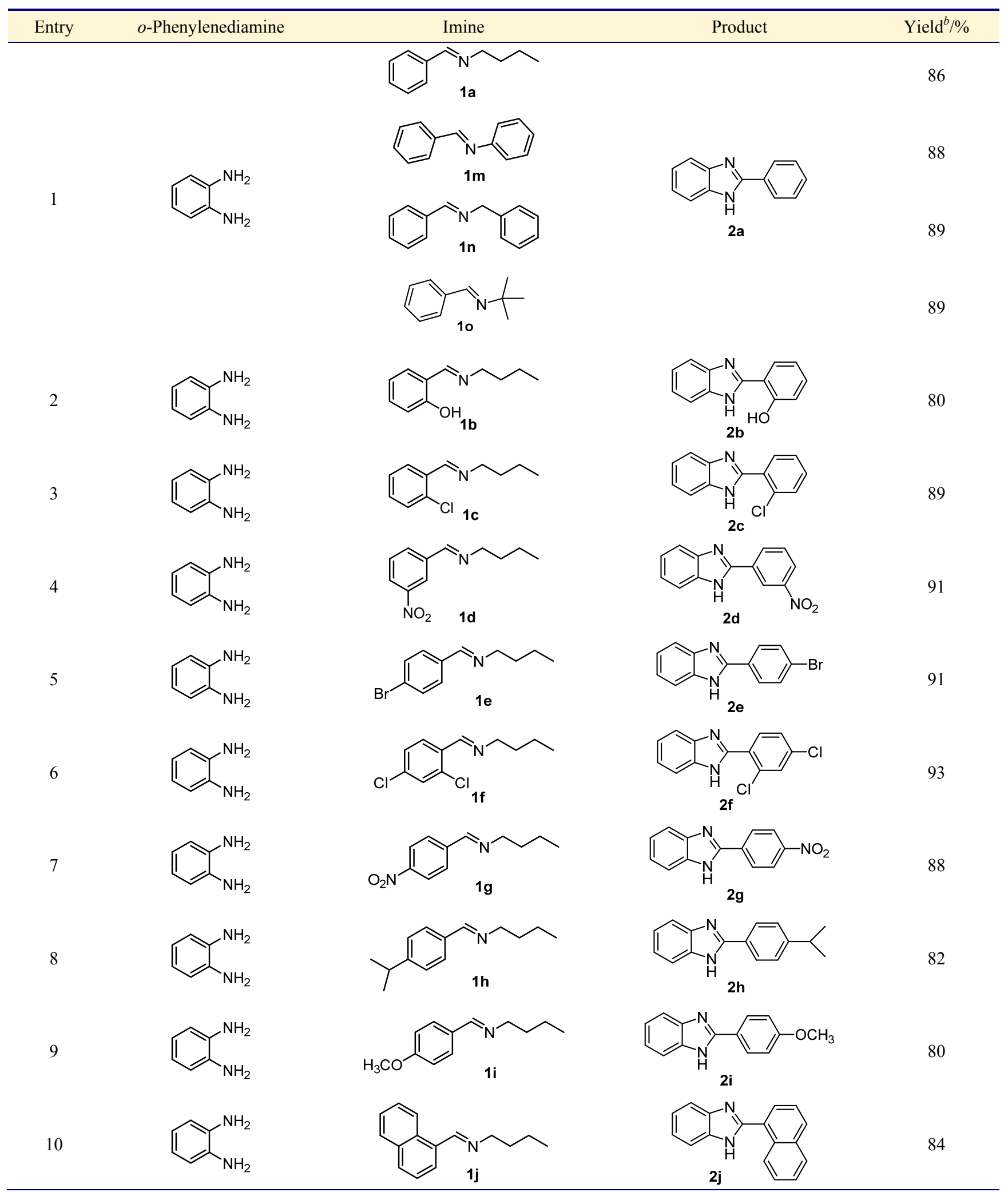




Entry

反应条件: 邻苯二胺 $(1.5 \mathrm{mmol})$, 亚胺 $(1.0 \mathrm{mmol}), \mathrm{Fe}\left(\mathrm{NO}_{3}\right)_{3} \cdot{ }^{\circ} \mathrm{H}_{2} \mathrm{O}(5 \mathrm{~mol} \%)$, 乙腈 $(5 \mathrm{~mL}), 80{ }^{\circ} \mathrm{C}$. 空气氛围, $10 \mathrm{~h} ;{ }^{b}$ 分离产率.

表 3 空气氛围下 $\mathrm{Fe}\left(\mathrm{NO}_{3}\right)_{3} \cdot 9 \mathrm{H}_{2} \mathrm{O}$ 催化苯胺和亚胺的有氧氧化 生成 $\mathrm{N}$-苯亚甲基苯胺 ${ }^{a}$

Table $3 \mathrm{Fe}\left(\mathrm{NO}_{3}\right)_{3} \cdot 9 \mathrm{H}_{2} \mathrm{O}$ catalyzed aerobic oxidation of aniline and imine to N-benzylidene-aniline under air

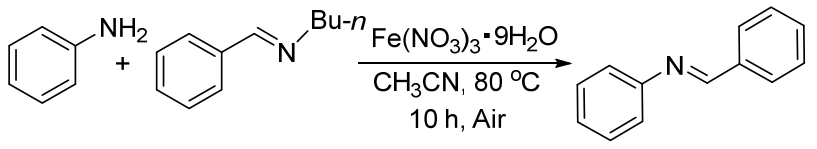

\begin{tabular}{cccc}
\hline Entry & Catalyst $(\mathrm{mol} \%)$ & Gas & Yield $^{b} \%$ \\
\hline 1 & $\mathrm{Fe}\left(\mathrm{NO}_{3}\right)_{3} \bullet 9 \mathrm{H}_{2} \mathrm{O}(5)$ & Air & 90 \\
2 & - & Air & Trace \\
3 & $\mathrm{Fe}\left(\mathrm{NO}_{3}\right)_{3} \bullet 9 \mathrm{H}_{2} \mathrm{O}(5)$ & $\mathrm{N}_{2}$ & Trace \\
\hline
\end{tabular}

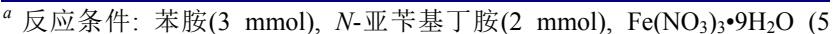
$\mathrm{mol} \%)$, 乙腈 $(5 \mathrm{~mL}), 80{ }^{\circ} \mathrm{C}$. 空气氛围, $10 \mathrm{~h} ;{ }^{b}$ 分离产率.

表 4 苯并咪唑啉环化脱氢生成苯并咪唑 ${ }^{a}$

Table 4 The cyclo-dehydrogenation of benzimidazoline to benzimidazole

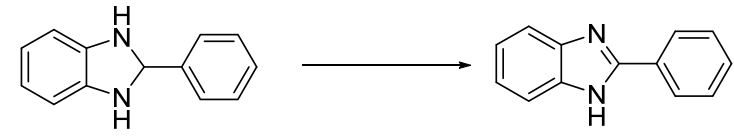

\begin{tabular}{cccc}
\hline Entry & Catalyst $(\mathrm{mol} \%)$ & Gas & Yield $^{b} \%$ \\
\hline 1 & $\mathrm{Fe}\left(\mathrm{NO}_{3}\right)_{3} \bullet 9 \mathrm{H}_{2} \mathrm{O}(5)$ & Air & 88 \\
2 & - & Air & 24 \\
3 & $\mathrm{Fe}\left(\mathrm{NO}_{3}\right)_{3} \bullet 9 \mathrm{H}_{2} \mathrm{O}(5)$ & $\mathrm{N}_{2}$ & Trace \\
\hline
\end{tabular}

反应条件: 邻苯二胺 $(1.0 \mathrm{mmol})$, 苯甲醛 $(1.0 \mathrm{mmol})$, 水 $(2.5 \mathrm{~mL})$, 乙醇 $(2.5$ $\mathrm{mL})$, r.t., $2 \mathrm{~h} ; \mathrm{Fe}\left(\mathrm{NO}_{3}\right)_{3} \cdot 9 \mathrm{H}_{2} \mathrm{O}(5 \mathrm{~mol} \%)$, 乙腈 $(5 \mathrm{~mL}), 80{ }^{\circ} \mathrm{C}$, 空气氛围, 10 $\mathrm{h},{ }^{b}$ 分离产率.

为溶剂, 在空气氛围下反应 $10 \mathrm{~h}$, 可以得到较高产率的 目标产物. 且反应过程采用空气作为氧化剂, 这为 2-取 代苯并咪唑化合物的制备提供了一条产品收率相对较 高的绿色合成途径. 另一方面初步探讨了该反应的合成

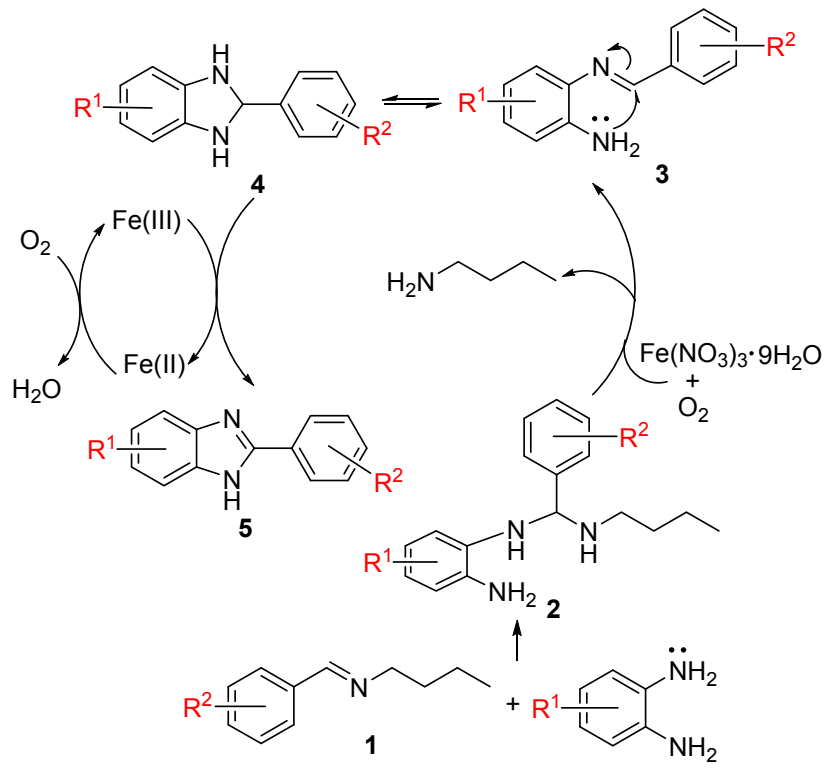

图 1 反应机理

Figure 1 Proposed reaction mechanism

机理. 该反应过程可分为三步: (1)亚胺和邻苯二胺氧化 缩合生成席夫碱; (2)席夫碱与邻苯二胺上另一氨基发生 亲核加成生成苯并咪唑啉; (3)苯并咪唑啉氧化脱氢生成 目标产物苯并咪唑.

\section{3 实验部分}

\section{1 仪器与试剂}

核磁共振仪: Bruker AM 500, Bruker AM 300(氛代 氯仿和氞代二甲亚峰为溶剂, TMS 为内标); 熔点仪: X-4 显微熔点仪(温度计未校正). 除亚胺自己合成外, 
剩余所用药品和试剂均为市售，未经进一步处理.

\section{2 实验方法}

在装有摚拌磁子、回流冷凝管的 $10 \mathrm{~mL}$ 圆底烧瓶中, 加入邻苯二胺 $(0.1628 \mathrm{~g}, 1.5 \mathrm{mmol}), N$-亚苄基丁胺(1a) (0.1613 g, $1.0 \mathrm{mmol}), \mathrm{Fe}\left(\mathrm{NO}_{3}\right)_{3} \cdot 9 \mathrm{H}_{2} \mathrm{O}(0.0212 \mathrm{~g}, 5 \mathrm{~mol} \%)$, 乙腈 $(5 \mathrm{~mL})$, 在 $80{ }^{\circ} \mathrm{C}$. 空气氛围下, 反应 $10 \mathrm{~h}$. 反应过 程中通过 TLC 对反应进行检测. 反应完成后, 反应混合 物浓缩, 粗产品通过柱层析分离纯化 $[V($ 乙酸乙酯)： $V($ 石油醚 $)=1: 8]$ 得到目标产物 $\mathbf{2 a}$.

2-苯基苯并咪唑(2a): 白色固体, m.p. 293 295 ${ }^{\circ} \mathrm{C}$ (lit. ${ }^{[17]}$ m.p. $\left.295 \sim 297{ }^{\circ} \mathrm{C}\right) ;{ }^{1} \mathrm{H}$ NMR (500 MHz, DMSO- $d_{6}$ ) $\delta: 12.93(\mathrm{~s}, 1 \mathrm{H}), 8.19(\mathrm{~d}, J=7.5 \mathrm{~Hz}, 2 \mathrm{H}), 7.66(\mathrm{~s}, 1 \mathrm{H}), 7.55$ (t, $J=7.0,8.0 \mathrm{~Hz}, 3 \mathrm{H}), 7.55(\mathrm{t}, J=7.0,7.5 \mathrm{~Hz}, 1 \mathrm{H}), 7.21$ (d, $J=4.0 \mathrm{~Hz}, 2 \mathrm{H})$.

2-(2-羟基苯基)苯并咪唑 (2b): 黄色固体, m.p. $241 \sim 242{ }^{\circ} \mathrm{C}$ (lit. ${ }^{[18]}$ m.p. $\left.240 \sim 242{ }^{\circ} \mathrm{C}\right) ;{ }^{1} \mathrm{H}$ NMR $(500$ MHz, DMSO- $\left.d_{6}\right) \delta: 13.19(\mathrm{~s}, 1 \mathrm{H}), 13.15(\mathrm{~s}, 1 \mathrm{H}), 8.05$ (dd, $J=1.5,1.5 \mathrm{~Hz}, 1 \mathrm{H}), 7.72(\mathrm{~d}, J=7.5 \mathrm{~Hz}, 1 \mathrm{H}), 7.61$ (d, $J=$ $7.5 \mathrm{~Hz}, 1 \mathrm{H}), 7.40 \sim 7.37(\mathrm{~m}, 1 \mathrm{H}), 7.31 \sim 7.27(\mathrm{~m}, 2 \mathrm{H})$, $7.05 \sim 7.01(\mathrm{~m}, 2 \mathrm{H})$.

2-(2-氯苯基)苯并咪唑(2c)：白色固体，m.p. 234 $235{ }^{\circ} \mathrm{C}$ (lit. ${ }^{[19]}$ m.p. $\left.233 \sim 235{ }^{\circ} \mathrm{C}\right) ;{ }^{1} \mathrm{H}$ NMR $(500 \mathrm{MHz}$, DMSO- $\left.d_{6}\right) \delta: 12.72(\mathrm{~s}, 1 \mathrm{H}), 7.92 \sim 7.90(\mathrm{~m}, 1 \mathrm{H}), 7.70 \sim$ $7.66(\mathrm{~m}, 2 \mathrm{H}), 7.56 \sim 7.52(\mathrm{~m}, 3 \mathrm{H}), 7.26 \sim 7.22(\mathrm{~m}, 2 \mathrm{H})$.

2-(3-硝基苯基)苯并咪唑(2d)：黄色固体, m.p. 202 204 ${ }^{\circ} \mathrm{C}$ (lit. ${ }^{[20]}$ m.p. $203 \sim 204{ }^{\circ} \mathrm{C}$ ); ${ }^{1} \mathrm{H}$ NMR $(500$ MHz, DMSO- $\left.d_{6}\right) \delta: 13.29(\mathrm{~s}, 1 \mathrm{H}), 9.02(\mathrm{~s}, 1 \mathrm{H}), 8.62(\mathrm{~d}$, $J=8.0 \mathrm{~Hz}, 1 \mathrm{H}), 8.34(\mathrm{dd}, J=1.5,1.5 \mathrm{~Hz}, 1 \mathrm{H}), 7.86(\mathrm{t}, J=$ 8.0, $8.0 \mathrm{~Hz}, 1 \mathrm{H}), 7.73$ (d, $J=8.0 \mathrm{~Hz}, 1 \mathrm{H}), 7.59$ (d, $J=7.5$ $\mathrm{Hz}, 1 \mathrm{H}), 7.30 \sim 7.23(\mathrm{~m}, 2 \mathrm{H})$.

2-(4-溴苯基)苯并咪唑(2e)：白色固体, m.p. 297 $299{ }^{\circ} \mathrm{C}$ (lit. ${ }^{[21]}$ m.p. $\left.298 \sim 299{ }^{\circ} \mathrm{C}\right) ;{ }^{1} \mathrm{H}$ NMR $(500 \mathrm{MHz}$, DMSO- $\left.d_{6}\right) \delta: 12.99(\mathrm{~s}, 1 \mathrm{H}), 8.12(\mathrm{~d}, J=9.0 \mathrm{~Hz}, 2 \mathrm{H}), 7.76$ (d, $J=8.5 \mathrm{~Hz}, 2 \mathrm{H}), 7.64 \sim 7.58(\mathrm{~m}, 2 \mathrm{H}), 7.22(\mathrm{~d}, J=3.5$ $\mathrm{Hz}, 2 \mathrm{H})$.

2-(2,4-二氯苯基)苯并咪唑(2f): 黄色固体, m.p. $217 \sim 219{ }^{\circ} \mathrm{C}$ (lit. ${ }^{[22]}$ m.p. $218 \sim 220{ }^{\circ} \mathrm{C}$ ); ${ }^{1} \mathrm{H}$ NMR $(500$ MHz, DMSO- $\left.d_{6}\right) \delta: 12.76(\mathrm{~s}, 1 \mathrm{H}), 7.94(\mathrm{~d}, J=8.5 \mathrm{~Hz}, 1 \mathrm{H})$, 7.85 (s, 1H), 7.70 (d, $J=7.5 \mathrm{~Hz}, 1 \mathrm{H}), 7.62(\mathrm{~d}, J=8.5 \mathrm{~Hz}$, $1 \mathrm{H}), 7.58(\mathrm{~d}, J=7.5 \mathrm{~Hz}, 1 \mathrm{H}), 7.27 \sim 7.23(\mathrm{~m}, 2 \mathrm{H})$.

2-(4-硝基苯基)苯并咪唑(2g)：淡黄色固体，m.p. $321 \sim 323{ }^{\circ} \mathrm{C}$ (lit. ${ }^{[23]}$ m.p. $321 \sim 322{ }^{\circ} \mathrm{C}$ ); ${ }^{1} \mathrm{H}$ NMR $(500$ $\mathrm{MHz}$, DMSO- $\left.d_{6}\right) \delta: 13.29(\mathrm{~s}, 1 \mathrm{H}), 8.42 \sim 8.41(\mathrm{~m}, 4 \mathrm{H})$, $7.73(\mathrm{~d}, J=7.5 \mathrm{~Hz}, 1 \mathrm{H}), 7.59(\mathrm{~d}, J=8.0 \mathrm{~Hz}, 1 \mathrm{H}), 7.31 \sim$ $7.24(\mathrm{~m}, 2 \mathrm{H})$. 2-(4-异丙基苯基)苯并咪唑(2h)：淡黄色固体，m.p. 251 253 ${ }^{\circ} \mathrm{C}$ (lit. ${ }^{[24]}$ m.p. $250 \sim 251{ }^{\circ} \mathrm{C}$ ); ${ }^{1} \mathrm{H}$ NMR $(500$ MHz, DMSO- $\left.d_{6}\right) \delta: 12.82(\mathrm{~s}, 1 \mathrm{H}), 8.10(\mathrm{~d}, J=5.0 \mathrm{~Hz}, 2 \mathrm{H})$, $7.65(\mathrm{~d}, J=7.5 \mathrm{~Hz}, 1 \mathrm{H}), 7.51(\mathrm{~d}, J=7.5 \mathrm{~Hz}, 1 \mathrm{H}), 7.42(\mathrm{~d}$, $J=8.0 \mathrm{~Hz}, 2 \mathrm{H}), 7.20 \sim 7.17(\mathrm{~m}, 2 \mathrm{H}), 3.00 \sim 2.93(\mathrm{~m}, 1 \mathrm{H})$, $1.25(\mathrm{~d}, J=7.0 \mathrm{~Hz}, 6 \mathrm{H})$.

2-(4-甲氧基苯基)苯并咪唑(2i)：淡黄色固体, m.p. $227 \sim 229{ }^{\circ} \mathrm{C}$ (lit. ${ }^{[25]}$ m.p. $\left.228 \sim 230{ }^{\circ} \mathrm{C}\right) ;{ }^{1} \mathrm{H}$ NMR $(500$ MHz, DMSO- $\left.d_{6}\right) \delta: 12.73(\mathrm{~s}, 1 \mathrm{H}), 8.11(\mathrm{~d}, J=9.0 \mathrm{~Hz}, 2 \mathrm{H})$, $7.62(\mathrm{~d}, J=7.5 \mathrm{~Hz}, 1 \mathrm{H}), 7.49(\mathrm{~d}, J=7.5 \mathrm{~Hz}, 1 \mathrm{H}), 7.18 \sim$ $7.15(\mathrm{~m}, 2 \mathrm{H}), 7.11(\mathrm{~d}, J=9.0 \mathrm{~Hz}, 2 \mathrm{H}), 3.84(\mathrm{~s}, 3 \mathrm{H})$.

2-(1-荎基)苯并咪唑 $(\mathbf{2 j})$ ：淡黄色固体, m.p. 269 $272{ }^{\circ} \mathrm{C}$ (lit. ${ }^{[26]}$ m.p. $270 \sim 272{ }^{\circ} \mathrm{C}$ ); ${ }^{1} \mathrm{H}$ NMR $(500 \mathrm{MHz}$, DMSO- $\left.d_{6}\right) \delta: 12.92(\mathrm{~s}, 1 \mathrm{H}), 9.10(\mathrm{~d}, J=8.5 \mathrm{~Hz}, 1 \mathrm{H}), 8.10$ (d, $J=8.5 \mathrm{~Hz}, 1 \mathrm{H}), 8.05 \sim 8.01(\mathrm{~m}, 2 \mathrm{H}), 7.78(\mathrm{~d}, J=7.5$ $\mathrm{Hz}, 1 \mathrm{H}), 7.70 \sim 7.58(\mathrm{~m}, 4 \mathrm{H}), 7.30 \sim 7.23(\mathrm{~m}, 2 \mathrm{H})$.

5-硝基-2-苯基苯并咪唑(2k): 红色固体, m.p. 207 $209{ }^{\circ} \mathrm{C}$ (lit. ${ }^{[27]}$ m.p. $\left.208 \sim 210{ }^{\circ} \mathrm{C}\right) ;{ }^{1} \mathrm{H}$ NMR $(300 \mathrm{MHz}$, DMSO- $\left.d_{6}\right) \delta: 13.59(\mathrm{~s}, 1 \mathrm{H}), 8.47(\mathrm{~s}, 1 \mathrm{H}), 8.24 \sim 8.11(\mathrm{~m}$, $3 \mathrm{H}), 7.59 \sim 7.47(\mathrm{~m}, 4 \mathrm{H})$.

5-氯-2-苯基苯并咪唑(2I)：白色固体, m.p. 212 $214{ }^{\circ} \mathrm{C}$ (lit. ${ }^{[27]}$ m.p.212 $214{ }^{\circ} \mathrm{C}$ ); ${ }^{1} \mathrm{H}$ NMR $(300 \mathrm{MHz}$, DMSO- $\left.d_{6}\right) \delta: 13.09(\mathrm{~s}, 1 \mathrm{H}), 8.15(\mathrm{~d}, J=7.5 \mathrm{~Hz}, 2 \mathrm{H})$, $7.70 \sim 7.50(\mathrm{~m}, 5 \mathrm{H}), 7.23(\mathrm{~s}, 1 \mathrm{H})$.

2-(3-吲哚基)苯并咪唑 (2m): 淡黄色固体, m.p. 195 $197{ }^{\circ} \mathrm{C}$ (lit. ${ }^{[27]}$ m.p. $196 \sim 198{ }^{\circ} \mathrm{C}$ ); ${ }^{1} \mathrm{H}$ NMR $(300$ MHz, DMSO- $\left.d_{6}\right) \delta: 11.22(\mathrm{~s}, 1 \mathrm{H}), 10.64(\mathrm{~s}, 1 \mathrm{H}), 8.32(\mathrm{~s}$, $1 \mathrm{H}), 7.80 \sim 7.73(\mathrm{~m}, 1 \mathrm{H}), 7.54 \sim 7.30(\mathrm{~m}, 3 \mathrm{H}), 7.23 \sim 6.86$ $(\mathrm{m}, 4 \mathrm{H})$.

2-(2-吡啶基)苯并咪唑(2n)：米白色固体，m.p. $217 \sim 219{ }^{\circ} \mathrm{C}$ (lit. ${ }^{[28]}$ m.p. $219 \sim 220{ }^{\circ} \mathrm{C}$ ); ${ }^{1} \mathrm{H}$ NMR $(300$ MHz, DMSO- $\left.d_{6}\right) \delta: 13.08(\mathrm{~s}, 1 \mathrm{H}), 8.72(\mathrm{~d}, J=4.2 \mathrm{~Hz}, 1 \mathrm{H})$, $8.31(\mathrm{~d}, J=7.8 \mathrm{~Hz}, 1 \mathrm{H}), 7.98(\mathrm{t}, J=7.8,7.8 \mathrm{~Hz}, 1 \mathrm{H}), 7.68$ (d, $J=7.8 \mathrm{~Hz}, 1 \mathrm{H}), 7.51(\mathrm{t}, J=6.9,6.9 \mathrm{~Hz}, 2 \mathrm{H}), 7.21(\mathrm{~m}$, $2 \mathrm{H})$.

辅助材料(Supporting Information) 亚胺合成、亚胺 ${ }^{1} \mathrm{H}$ NMR 谱图、中间体及苯并咪唑衍生物 ${ }^{1} \mathrm{H}$ NMR 谱图. 这 些材料可以免费从本刊网站(http://sioc-journal.cn/)上下 载.

\section{References}

[1] Li, Y.-Y.; Zhou, Y.-H.; Guo, Y.-F. Chin. J. Org. Chem. 2006, 26, 1097 (in Chinese).

(李莹莹, 周永花, 郭玉芳, 有机化学, 2006, 26, 1097.)

[2] [2] Lu, J.; Ge, H.-G.; Bai, Y.-J. Chin. J. Org. Chem. 2002, 22, 782 (in Chinese). 
(路军, 葛红光, 白银娟, 有机化学, 2002, 22, 782.)

[3] Mao, Z.-Z.; Wang, Z.-Y.; Hou, X.-N.; Song, X.-M.; Luo, Y.-F. Chin. J. Org. Chem. 2008, 28, 542 (in Chinese).

(毛郑州, 汪朝阳, 侯晓娜, 宋秀美, 罗玉芬, 有机化学, 2008, 28, 542.)

[4] Ke, F.; Xu, J.-H.; Liu, C.-Q.; Li, Q.; Wu, W.; Li, P. Chin. J. Org. Chem. 2014, 34, 1900 (in Chinese).

(柯方, 许建华, 刘彩琴, 黎迁, 吴雯, 李鹏, 有机化学, 2014, 34, 1900.)

[5] Bahrami, K.; Khodaei, M. M.; Nejati, A. Green Chem. 2010, 12, 1237.

[6] Bahrami, K.; Khodaei, M. M.; Kavianinia, I. Synthesis 2007, 547.

[7] (a) Sun, C.-L.; Li, B.-J.; Shi, Z.-J. Chem. Rev. 2011, 111, 1293.

(b) Gopalaiah, K. Chem. Rev. 2013, 113, 3248.

(c) Zhu, S.-F.; Zhou, Q.-L. Nat. Sci. Rev. 2014, 1, 580.

(d) Bauer, I.; Knölker, H.-J. Chem. Rev. 2015, 115, 3170.

(e) Guo, N.; Zhu, S.-F. Chin. J. Org. Chem. 2015, 35, 1383 (in Chinese).

(郭娜, 朱守非, 有机化学, 2015, 35, 1383.)

[8] Guo, X.-G.; Fang, G.-Z.; Li, G.; Ma, H.; Fan, H.-J.; Yu, L.; Ma, C.; Wu, X.; Deng, D.-H.; Wei, M.-M.; Tan, D.-L.; Si, R.; Zhang, S.; Li, J.-Q.; Sun, L.-T.; Tang, Z.-C.; Pan, X.-L.; Bao, X.-H. Science 2014, $344,616$.

[9] Li, Y.-Y.; Yu, S.-L.; Wu, X.-F.; Xiao, J.-L.; Shen, W.-Y.; Dong, Z.-R.; Gao, J.-X. J. Am. Chem. Soc. 2014, 136, 4031.

[10] Shen, J.-J.; Zhu, S.-F., Cai, Y.; Xu, H.; Xie, X.-L.; Zhou, Q.-L. Angew. Chem., Int. Ed. 2014, 53, 13188.

[11] Zhang, Y.; Luo, S.; Feng, B.-N. Chin. J. Org. Chem. 2014, 34, 2249 (in Chinese).

(张艳, 罗莎, 冯柏年, 有机化学, 2014, 34, 2249.)
[12] Liu, S.-S.; Jiang, K.; Pi, D.-W.; Zhou, H.-F.; Uozumi, Y.; Zou, K. Chin. J. Org. Chem. 2014, 34, 1369 (in Chinese).

(刘森生, 姜坤, 皮单违, 周海峰, Yasuhiro Uozumi, 邹坤, 有机 化学, 2014, 34, 1369.)

[13] Guo, N.; Hu, M.-Y.; Feng, Y.; Zhu, S.-F. Org. Chem. Front. 2015, 2, 692.

[14] Yu, J.-T.; Xu, J.; Lu, M. Appl. Organomet. Chem. 2013, 27, 606.

[15] Yu, J.-T.; Lu, M. Synth. Commun. 2014, 44, 2520.

[16] Yu, J.-T.; Xia, Y.-G.; Lu, M. Synth. Commun. 2014, 44, 3019.

[17] Chari, M. A.; Shobha, D.; Sasaki, T. Tetrahedron Lett. 2011, 52, 5575.

[18] Sharghi, H.; Beyzavi, M. H.; Doroodmand, M. M. Eur. J. Org. Chem. 2008, 24, 4126.

[19] Behbahani, F. K.; Ziaei, P.; Fakhroueian, Z.; Doragi, N. Monatsh. Chem. 2011, 142, 901.

[20] Han, X.-M.; Ma, H.-Q.; Wang, Y.-L. Russ. J. Org. Chem. 2008, 44, 863.

[21] Shen, M.-H.; Driver, T. G. Org. Lett. 2008, 10, 3367.

[22] Chari, M. A.; Shobha, D. Tetrahedron Lett. 2010, 51, 5195.

[23] Maraš, N.; Kočevar, M. Helv. Chim. Acta 2011, 94, 1874.

[24] Sharghi, H.; Beyzavi, M. H.; Doroodmand, M. M. Eur. J. Org. Chem. 2008, 24, 4138.

[25] Ma, H.-Q.; Wang, Y.-L.; Wang, J.-Y. Heterocycles 2006, 68, 1669.

[26] Joshi, R. S.; Mandhane, P. G.; Dabhade, S. K.; Gill, C. J. Chin. Chem. Soc. 2010, 57, 1231.

[27] Chari, M. A.; Shobha, D.; Sasaki, T. Tetrahedron Lett. 2011, 52, 5575.

[28] Nguyen, T. B.; Ermolenko, L.; Dean, W. A.; Al-Mourabit, A. Org. Lett. 2012, 14, 5948. 